\title{
Remoción del colorante AV7 presente en solución acuosa mediante carbón activado
}

\author{
Emmanuel Bretón Jiménez ${ }^{A}$, Edwin Javier Melgar Urbina ${ }^{B}$, \\ Miguel Ángel Hernández RiveraA, Margarita Loredo Cancino ${ }^{\mathrm{B}}$, \\ Nancy Elizabeth Dávila Guzmán*B \\ AUniversidad Juárez Autónoma de Tabasco, Cunduacán, México. \\ ${ }^{B}$ Universidad Autónoma de Nuevo León, Facultad de Ciencias Químicas, \\ Departamento de Ingeniería Química \\ nancy.davilagz@uanl.edu.mx
}

\begin{abstract}
RESUMEN
Se investigó la remoción del colorante ácido violeta 7 mediante carbón activado en un sistema de adsorción en lote. Se estudiaron los efectos de parámetros experimentales en la capacidad de adsorción, tales como la masa del adsorbente y el $\mathrm{pH}$. Las mejores condiciones experimentales para la adsorción de ácido violeta 7 fueron a $\mathrm{pH}$ natural del colorante ( $\mathrm{pH}$ 6) y $100 \mathrm{mg}$ de adsorbente al obtener una capacidad de adsorción de $102 \mathrm{mg} / \mathrm{g}$. La cinética de adsorción fue descrita por el modelo de Pseudo-Segundo orden que está basado en un mecanismo de quimisorción.
\end{abstract}

\section{PALABRAS CLAVE}

Adsorción; carbón activado; cinética; remoción de colorante.

\begin{abstract}
The removal of the acid violet dye 7 by activated carbon was investigated in a batch adsorption system. The effects of experimental parameters on the adsorption capacity, such as the mass of the adsorbent and the $\mathrm{pH}$ were studied. The best experimental conditions for the adsorption of acid violet 7 were at the natural $\mathrm{pH}$ of the dye $(\mathrm{pH} \mathrm{6})$ and $100 \mathrm{mg}$ of adsorbent achieving an adsorption capacity of $102 \mathrm{mg} / \mathrm{g}$. The adsorption kinetics followed the Pseudo-Second order model which is based on a quimisorption mechanism.
\end{abstract}

\section{KEYWORDS}

Adsorption; activated carbon; kinetics; dye removal.

\section{INTRODUCCIÓN}

El rápido crecimiento demográfico y desarrollo económico de la sociedad humana incrementan las actividades industriales y agrícolas, haciendo un uso acelerado de agua en todo el mundo. Se prevé que en el año 2050 la población mundial alcance 9,800 millones de habitantes que podrían llegar a usar hasta un $55 \%$ más de agua para satisfacer las necesidades básicas en todo el mundo. ${ }^{1}$ Los recursos hídricos han sido explotados de manera descontrolada por industrias tales como la textil, la papelera, la farmacéutica, la alimentaria y la cosmética, 
siendo el color la primera evidencia de la contaminación generada. ${ }^{2}$ Alrededor de 800,000 toneladas de tintes se producen anualmente en el mundo y el 60-70 $\%$ son colorantes. Esto genera un gran impacto ambiental que contaminan las masas de agua con facilidad, repercutiendo en la salud humana, en las tierras de cultivo y en la actividad acuícola. ${ }^{3}$ Los colorantes azoicos son compuestos que tienen el grupo azo cromóforo $\left(\mathrm{R}_{1}-\mathrm{N}=\mathrm{N}-\mathrm{R}_{2}\right)$ en sus estructuras moleculares. Estos grupos funcionales dificultan la biodegradación de los mismos y se estima que entre el 10 y $15 \%$ de estos compuestos se pierde y se incorpora en el efluente industrial, los cuales deberían ser eliminados antes de ser descargados a las masas de agua, debido a su alta toxicidad y contaminación provocada en los ecosistemas. ${ }^{4}$ El colorante ácido violeta 7 (AV7) es muy importante en la industria textil y uno de los mayores contribuyentes de la contaminación, ${ }^{5}$ este colorante está formado por grupos aromáticos complejos y sulfónicos que lo hacen altamente genotóxico comparado con otros colorantes azoicos. Los colorantes azoicos afectan a la salud humana, y al medio ambiente. En los últimos años, algunos científicos han tratado de resolver esta problemática. Entre los diversos métodos de eliminación comúnmente utilizados destacan la adsorción, foto-degradación y decoloración microbiológica, entre otros. ${ }^{6}$ En general, la adsorción de colorantes de la industria textil sobre materiales sólidos como el carbón activado (CA) resulta eficiente gracias a la relativa simplicidad del sistema y a las propiedades del carbón activado, como elevada área superficial, estabilidad química y alta capacidad de adsorción. ${ }^{7}$ El CA ha sido utilizado en la purificación del aire, remoción de colorantes y contaminantes farmacéuticos, entre otros. ${ }^{8}$ Por lo tanto, en esta investigación se evaluó la capacidad del CA para la remoción del contaminante AV7, a través del estudio de equilibrio y cinético del proceso de adsorción.

\section{MATERIALES Y MÉTODOS \\ Reactivos y materiales}

El carbón activado utilizado fue de origen mineral, con tamaño de partícula de 500-700 $\mu \mathrm{m}$ (Clarimex). El ácido violeta 7 fue provisto por Sigma-Aldrich en forma de polvo, tiene un peso molecular de $566.47 \mathrm{~g} / \mathrm{mol} \mathrm{y}$ su fórmula química es $\mathrm{C}_{20} \mathrm{H}_{16} \mathrm{~N}_{4} \mathrm{Na}_{2} \mathrm{O}_{9} \mathrm{~S}_{2}$.

\section{Preparación del adsorbato}

Una solución patrón de ácido violeta $7 \mathrm{de} 50 \mathrm{mg} / \mathrm{L}$ se preparó añadiendo $0.05 \mathrm{~g}$ de ácido violeta 7 sólido en un matraz volumétrico de $0.025 \mathrm{~L}$ con agua desionizada, agitándose hasta obtener una solución homogénea color violeta.

\section{Análisis de concentración del adsorbato.}

La concentración del adsorbato fue cuantificada en un espectrofotómetro (Genesis 10S UV/Vis) utilizando una curva de calibración. Los estándares del AV7 fueron preparados a partir de la solución patrón para obtener concentraciones de $1 \mathrm{mg} / \mathrm{L}, 2 \mathrm{mg} / \mathrm{L}, 5 \mathrm{mg} / \mathrm{L}, 10 \mathrm{mg} / \mathrm{L}$ y $25 \mathrm{mg} / \mathrm{L}$ Posteriormente, se registró la absorbancia de cada estándar a una longitud de onda de $510 \mathrm{~nm}$. 


\section{Experimentos de cinética de adsorción}

La cinética de adsorción de AV7 sobre CA fue obtenida añadiendo una masa definida de CA a un matraz Erlenmeyer de capacidad nominal de $250 \mathrm{~mL}$, la cual fue puesta en contacto con $100 \mathrm{~mL}$ de AV7 a una concentración inicial de $50 \mathrm{mg} / \mathrm{L}$. La mezcla se mantuvo en agitación constante a 200 rpm y a temperatura ambiente $\left(25^{\circ} \mathrm{C}\right)$ durante un tiempo de contacto de $6 \mathrm{~h}$ (para asegurar alcanzar el equilibrio). Se tomaron alícuotas de $1 \mathrm{~mL}$ de la solución a diferentes tiempos $(5,10,20$, $30,60,90,120,150,180,240$, y $360 \mathrm{~min}$ ) para su posterior análisis mediante espectrofotometría UV-Vis. La cantidad de colorante adsorbido en cualquier tiempo, q (mg/g), se calculó por medio del siguiente balance de masa:

$$
q=\frac{(C o-C) V}{m}
$$

Donde Co y $\mathrm{C}$ son las concentraciones iniciales y a cualquier tiempo de AV7 $(\mathrm{mg} / \mathrm{L})$, respectivamente; $\mathrm{V}$ es el volumen de la solución (L) y m es la masa del adsorbente $(\mathrm{g})$.

El porcentaje de remoción del adsorbente en el equilibrio fue calculado mediante la siguiente (ecuación 2):

Remoción $(\%)=\frac{(\mathrm{Co}-\mathrm{Ce})}{\mathrm{Co}} \times 100$

Donde Ce es la concentración de AV7 en el equilibrio (mg/L).

\section{Efecto de la masa del adsorbente}

La cantidad empleada del adsorbente CA es un parámetro muy importante en el estudio de adsorción ya que determina la capacidad del adsorbente para tratar una solución coloreada de una concentración inicial dada. Para el estudio del efecto de la masa del adsorbente se realizaron experimentos con tres niveles $(100,200$ y $300 \mathrm{mg})$ al $\mathrm{pH}$ natural del colorante; a una concentración inicial de $50 \mathrm{mg} / \mathrm{L}$ de AV7 (tabla I). Es importante mencionar que el $\mathrm{pH}$ no fue controlado durante los experimentos de adsorción.

\section{Efecto del pH sobre la capacidad de adsorción}

El pH de la solución es un parámetro muy importante en el proceso de adsorción ya que puede afectar la interacción de los grupos funcionales superficiales del adsorbato y el adsorbente, además, las propiedades del CA y de los colorantes podrán indicar si la adsorción es favorable en disoluciones ácidas o básicas. ${ }^{9} \mathrm{El}$ efecto del $\mathrm{pH}$ sobre la capacidad de adsorción se evaluó en tres niveles de $\mathrm{pH}$ (3, 5 y 7), a una concentración inicial de $50 \mathrm{mg} / \mathrm{L}$ de AV7 (tabla II). El pH fue controlado durante el proceso de adsorción, mediante la adición de alícuotas de $\mathrm{HNO}_{3}$ y NaOH al $0.1 \mathrm{~N}$ según el caso.

Tabla I. Condiciones experimentales para evaluar el efecto de la masa de CA sobre la capacidad de adsorción AV7.

\begin{tabular}{|c|c|c|c|}
\hline Experimentos & CA-100 & CA-200 & CA-300 \\
\hline pH inicial & 6 & 6 & 6 \\
\hline Masa CA $(\mathrm{mg})$ & 100 & 200 & 300 \\
\hline
\end{tabular}




\section{Modelación matemática de la cinética de adsorción}

Los modelos de Pseudo-Primer orden y Pseudo-Segundo orden fueron empleados para la modelación matemática de las cinéticas de adsorción de AV7 en carbón activado. El modelo de Pseudo-Primer orden supone que la velocidad de adsorción con respecto al tiempo es proporcional a la diferencia entre la capacidad de adsorción en el equilibrio $\left(\mathrm{q}_{\mathrm{e}}\right)$ y la cantidad adsorbida en un tiempo determinado $\left(\mathrm{q}_{\mathrm{t}}\right)$, tal como se muestra en la (ecuación 3$)$.

$$
d \frac{q_{t}}{d t}=k_{1}\left(q_{e}-q_{t}\right)
$$

Al integrar la Ec. 3 en $\mathrm{q}(0)=0$ y $\mathrm{q}(\mathrm{t})=\mathrm{qt}$ se obtiene la Ec. 4 :

$$
\ln \left(q_{e}-q_{t}\right)=\ln q_{e}-k_{1} t
$$

Por otro lado, el modelo de Pseudo-Segundo orden supone que el paso limitante de la velocidad corresponde a la etapa de quimisorción, tal como se muestra en la Ec. 5:

$$
d \frac{q_{t}}{d t}=k_{2}\left(q_{e}-q_{t}\right)^{2}
$$

Integrando en $\mathrm{q}(0)=0$ y $\mathrm{q}(\mathrm{t})=\mathrm{qt}$ se obtiene la Ec. 6 :

Tabla II. Condiciones experimentales para evaluar el efecto del pH sobre la capacidad de adsorción de AV7.

\begin{tabular}{|c|c|c|c|}
\hline Experimentos & CA-3 & CA-5 & CA-7 \\
\hline $\mathrm{pH}$ & 3 & 5 & 7 \\
\hline Masa CA $(\mathrm{mg})$ & 100 & 100 & 100 \\
\hline
\end{tabular}

$$
\frac{t}{q_{t}}=\frac{1}{k_{2} q_{e}^{2}}+\frac{t}{q_{e}}
$$

Los parámetros de los modelos cinéticos fueron obtenidos mediante la minimización de la suma de los errores al cuadrado (Ec. 7) utilizando la herramienta Solver ${ }^{\circledR}$ de Microsoft Excel. Por otra parte, el coeficiente de determinación $\left(\mathrm{R}^{2}\right)$ fue calculado con la (Ec. 8).

$$
\begin{aligned}
& S E C=\sqrt{\frac{\left(q_{\text {exp }}-q_{c a l}\right)^{2}}{n-(k+1)}} \\
& R^{2}=\frac{\sum\left(q_{c a l}-\bar{q}_{c a l}\right)^{2}}{\sum\left(q_{c a l}-\bar{q}_{\text {exp }}\right)^{2}+\sum\left(q_{c a l}-q_{\text {exp }}\right)^{2}}
\end{aligned}
$$

Donde $q_{\text {cal }}, q_{\exp } \mathrm{y} \bar{q}_{\exp }$ son la capacidad de adsorción calculada con el modelo cinético, la capacidad de adsorción experimental, y el promedio de la capacidad de adsorción experimental, respectivamente, en $\mathrm{mg} / \mathrm{g}$, $\mathrm{n}$ es el número de datos experimentales y $k$ es el número de los parámetros del modelo.

\section{RESULTADOS Y DISCUSION}

\section{Efecto de la masa sobre la capacidad de adsorción}

Se evaluó la capacidad de adsorción de AV7 en CA a diferentes masas de 
adsorbente (figura 1). Los resultados muestran que conforme la cantidad de masa aumenta de 100 a $300 \mathrm{mg}$ de CA, la capacidad de adsorción disminuye de 102 a $33.8 \mathrm{mg} / \mathrm{g}$. Por otra parte, con el incremento de la masa de CA se observó un menor tiempo para alcanzar el equilibrio al pasar de 240 a 180 min para el experimento con CA-100 y CA-300, respectivamente. Estos resultados pueden ser atribuidos a que conforme se incrementa la cantidad de adsorbente se aumenta la cantidad de sitios de adsorción con lo que se favorece la velocidad de adsorción de AV7.

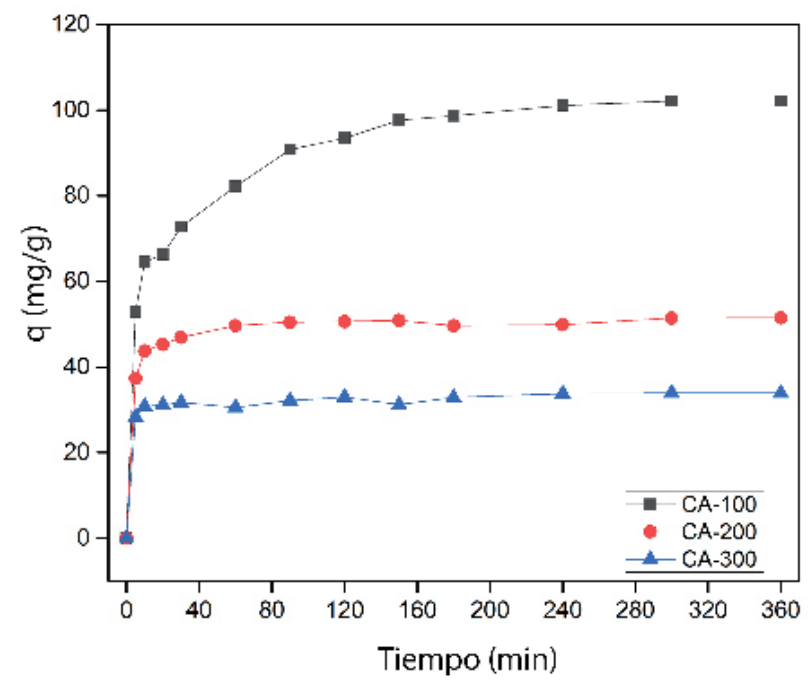

Fig. 1. Efecto de la masa sobre la cinética de adsorción. Condiciones: pH (6) natural del colorante, $25^{\circ} \mathrm{C}, \mathrm{Co}=50 \mathrm{mg} / \mathrm{L}$.

Por otra parte, el porcentaje de remoción de AV7 aumentó de 79.3\% a 91.6\% para un incremento de la masa de adsorbente de 100 a $300 \mathrm{mg}$ (figura 2). En conclusión, los sitios de adsorción disponibles para la remoción de AV7 dependen de la cantidad de adsorbente añadida a la solución. ${ }^{10}$

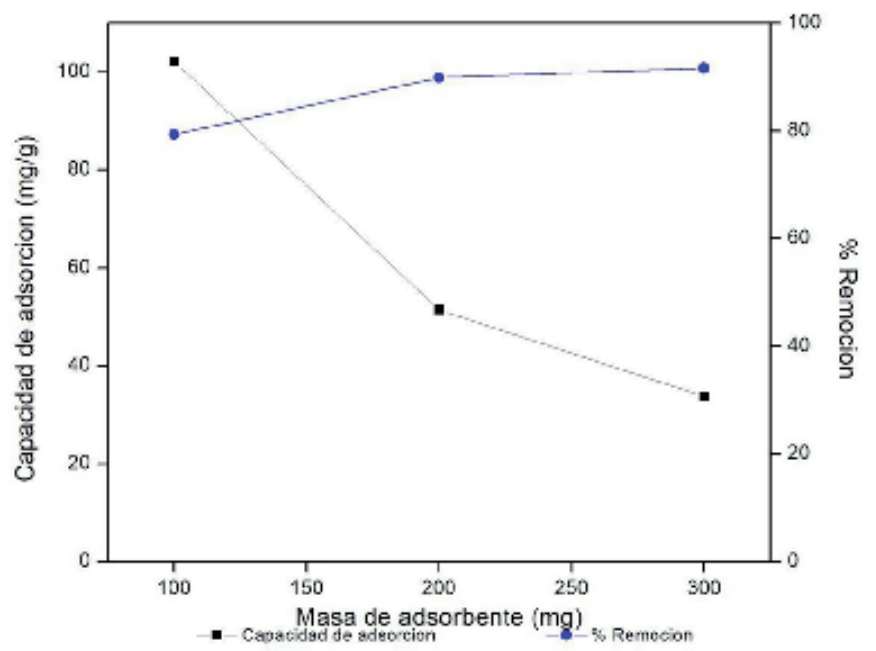

Fig. 2. Efecto de la dosis de masa de adsorbente en el porcentaje de remoción. 


\section{Efecto del pH sobre la capacidad de adsorción}

En la figura 3, muestra como disminuye la capacidad de adsorción del CA cuando se aumenta el pH de la solución. A pH 5 se obtuvo la mayor capacidad de adsorción $(86.6 \mathrm{mg} / \mathrm{g})$ mientras que a $\mathrm{pH} 7$ la capacidad de adsorción fue $61.4 \mathrm{mg} / \mathrm{g}$. A pH ácidos (3 a 5) la concentración de iones hidrógeno (H+) aumenta, cargándose positivamente la superficie del CA, situación que facilita las atracciones electrostáticas con las moléculas del colorante AV7 de condición aniónica. Se ha demostrado que a un $\mathrm{pH}$ ácido es posible que se formen centros electrofílicos en el CA que pueden generar atracciones de naturaleza electrostática con adsorbatos aniónicos. ${ }^{11}$

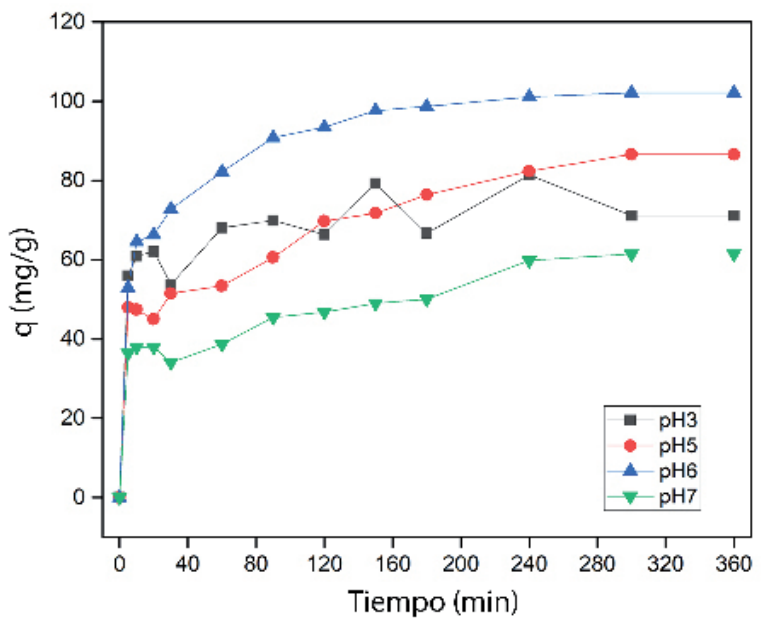

Fig. 3. Efecto del pH sobre la cinética de adsorción de AV7. Condiciones: $\mathrm{pH}(3,5,7)$ controlado durante todo el experimento y $\mathrm{pH}(6)$ sin control, $25^{\circ} \mathrm{C}$, Co de $50 \mathrm{mg} / \mathrm{L}$ y $100 \mathrm{mg}$ de CA.

\section{Modelación matemática de la cinética de adsorción}

Los datos de cinética de adsorción fueron ajustados a los modelos de Pseudoprimer orden y Pseudo-segundo orden (figura 4). Se encontró que el modelo de Pseudo-segundo orden es el modelo que mejor predice la cinética de adsorción de AV7 en CA, considerando la magnitud del error obtenido. De acuerdo al modelo de Pseudo-segundo orden, el proceso de adsorción está controlado por un mecanismo de quimisorción. Los parámetros de los modelos cinéticos obtenidos se encuentran reportados en la tabla III.

Tabla III. Parámetros cinéticos de los modelos de pseudo-primer orden y pseudo-segundo orden en la adsorción de AV7 sobre CA.

\begin{tabular}{|c|c|}
\hline \multicolumn{2}{|c|}{ Pseudo-Primer orden } \\
\hline $\mathrm{K}_{1}\left(\mathrm{~min}^{-1}\right)$ & 0.072 \\
\hline $\mathrm{R}^{2}$ & 0.891 \\
\hline $\mathrm{SEC}$ & 1656.12 \\
\hline \multicolumn{2}{|c|}{ Pseudo-Segundo orden } \\
\hline $\mathrm{K}_{2}\left(\mathrm{~g} \mathrm{mg}^{-1} \mathrm{~min}^{-1}\right)$ & 0.001 \\
\hline $\mathrm{R}^{2}$ & 0.958 \\
\hline $\mathrm{SEC}$ & 434.53 \\
\hline
\end{tabular}




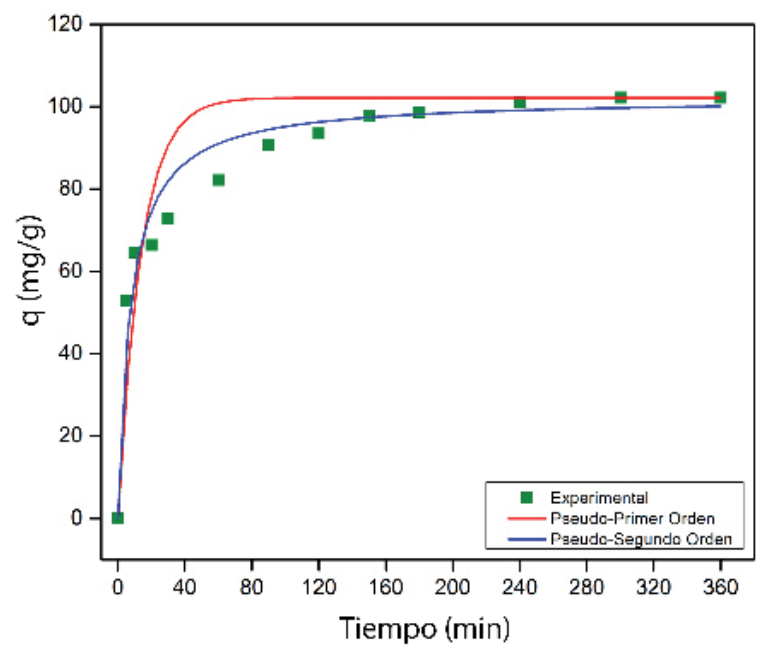

Fig. 4. Comparación de los modelos cinéticos de adsorción de pseudo-primer orden y pseudo-segundo orden para la remoción de AV7 con CA a $25^{\circ} \mathrm{C}$.

\section{CONCLUSIONES}

Se investigó el potencial del CA comercial como material adsorbente en la remoción de AV7 en soluciones acuosas. Se estudiaron los efectos de parámetros tales como masa del adsorbente, $\mathrm{pH}$ natural del colorante (6) y $\mathrm{pH}(3,5$ y 7). La mayor capacidad de adsorción fue obtenida en el experimento CA-100 con una masa de $100 \mathrm{mg}$ de CA con un valor de $102 \mathrm{mg} / \mathrm{g}$ a pH natural del colorante (6). La cinética de adsorción fue descrita por el modelo de Pseudo-segundo orden, el cual está basado en un mecanismo de quimisorción. Por lo tanto, este estudio demuestra que el adsorbente CA de origen comercial puede ser una alternativa eficiente para la remoción de colorantes azoicos en tratamientos terciarios de aguas residuales, además, la capacidad de adsorción aumentó al utilizar un $\mathrm{pH}$ natural (6), al no requerir la adición de más reactivos a la solución se puede reducir el costo de operación.

\section{REFERENCIAS}

1. Chen, L., Huang, K., Zhou, J., Duan, H. F., Zhang, J., Wang, D., \& Qiu, H. (2020). Multiple-risk assessment of water supply, hydropower and environment nexus in the water resources system. Journal of Cleaner Production, 268, 122057. https://doi.org/10.1016/j.jclepro.2020.122057.

2. Hincapié Mejía, G., Cardona Cuervo, S., \& Ríos, L. A. (2018). Absorption thermodynamic study of azoic dye with by means of a lignocellulosic waste in aqueous medium. Ingeniería y Desarrollo, 36(1), 97-118. https://doi. org/10.14482/inde.36.1.10941.

3. Ben Mansour, H., Corroler, D., Barillier, D., Ghedira, K., Chekir, L., \& Mosrati, R. (2007). Evaluation of genotoxicity and pro-oxidant effect of the azo dyes: Acids yellow 17, violet 7 and orange 52, and of their degradation products by Pseudomonas putida mt-2. Food and Chemical Toxicology, 45(9), 1670-1677. https://doi.org/10.1016/j.fct.2007.02.033. 
4. Moutaouakkil, A., Zeroual, Y., Dzayri, F. Z., Talbi, M., Lee, K., \& Blaghen, M. (2003). Purification and partial characterization of azoreductase from Enterobacter agglomerans. Archives of Biochemistry and Biophysics, 413(1), 139-146. https://doi.org/10.1016/S0003-9861(03)00096-1.

5. González-Casamachin, D. A., Rivera De la Rosa, J., Lucio-Ortiz, C. J., De Haro De Rio, D. A., Martínez-Vargas, D. X., Flores-Escamilla, G. A., Dávila Guzman, N. E., Ovando-Medina, V. M., \& Moctezuma-Velazquez, E. (2019). Visible-light photocatalytic degradation of acid violet 7 dye in a continuous annular reactor using $\mathrm{ZnO} / \mathrm{PPy}$ photocatalyst: Synthesis, characterization, mass transfer effect evaluation and kinetic analysis. Chemical Engineering Journal, 373(March), 325-337. https://doi.org/10.1016/j.cej.2019.05.032.

6. Forgacs, E., Cserháti, T., \& Oros, G. (2004). Removal of synthetic dyes from wastewaters: A review. Environment International, 30(7), 953-971. https://doi. org/10.1016/j.envint.2004.02.001.

7. Rafatullah, M., Sulaiman, O., Hashim, R., \& Ahmad, A. (2010). Adsorption of methylene blue on low-cost adsorbents: A review. Journal of Hazardous Materials, 177(1-3), 70-80. https://doi.org/10.1016/j.jhazmat.2009.12.047.

8. Oladejo, J., Shi, K., Chen, Y., Luo, X., Gang, Y., \& Wu, T. (2020). Closing the active carbon cycle: Regeneration of spent activated carbon from a wastewater treatment facility for resource optimization. Chemical Engineering and Processing - Process Intensification, 150(November 2019), 107878. https://doi.org/10.1016/j.cep.2020.107878.

9. Vargas, A. M. M., Cazetta, A. L., Martins, A. C., Moraes, J. C. G., Garcia, E. E., Gauze, G. F., Costa, W. F., \& Almeida, V. C. (2012). Kinetic and equilibrium studies: Adsorption of food dyes Acid Yellow 6, Acid Yellow 23, and Acid Red 18 on activated carbon from flamboyant pods. Chemical Engineering Journal, 181-182, 243-250. https://doi.org/10.1016/j.cej.2011.11.073.

10.Bhatti, H. N., Mumtaz, B., Hanif, M. A., \& Nadeem, R. (2007). Removal of $\mathrm{Zn}$ (II) ions from aqueous solution using Moringa oleifera Lam. (horseradish tree) biomass. Process Biochemistry, 42(4), 547-553. https://doi.org/10.1016/ j.procbio.2006.10.009.

11.Castelar Ortega, G. C., Viloria C, C. A., Morrinson B, C. A., Angulo M, E. R., \& Zambrano A, A. M. (2017). Evaluación de un carbón activado comercial en la remoción del colorante DB2. Revista Colombiana de Ciencia Animal - RECIA, 9(2), 164. https://doi.org/10.24188/recia.v9.n2.2017.512. 